\title{
Simulation Analysis of Negative-Pressure Chip Removing Device of Deep-Hole Processing Based on FLUENT Software
}

\author{
Zhen Dong, Fenghua Hao \\ North University of china, Taiyuan 030051, China \\ Paul Kwon \\ Joincheer Mechanical and Electrical Company, Seoul, Korea
}

\begin{abstract}
The negative-pressure chip removing device is a powerful tool to ensure chip removal smoothly in deep-hole processing. The paper establishes a mathematical model of the negative-pressure chip removing device, analyze the key factors which influencing the effect of pumping crumbs, simulated and contrast whether under the negative pressure pumping device cases or not by FLUENT software, to verify the pumping crumbs effects.
\end{abstract}

KEYWORD: Deep-hole processing; Negative-Pressure Chip Removing; FLUENT software emulation

\section{INTRODUCTION}

In recent years, with the rapid development of manufacturing industry, the low efficiency and accuracy of deep hole processing technology become a bottleneck for constraint entire manufacturing and assembly technology development. The impeded chip removing is one of the main problems affecting the development of deep - hole processing technology, especially in high-speed machining small deep hole, crumbs easily blocked, resulting in a high rejection rate. How to ensure the deep hole processing chip removal smooth, attracting more and more attention of the major research institutes and institutions. Currently, the conventional way to solve chip removal difficult in three ways: (1) expand the chip removal space; (2) control chip morphology; (3) enhance the chip removal power (SQ Wang, 2003).

Negative pressure chip removing technology is a way using the third chip removal pathway, increased the suction force, enabled proactive chip removal.

\section{THE WORKING PRINCIPLE OF NEGATIVE- PRESSURE CHIP REMOVING DEVICE}

As shown in Fig.1, the high pressure cutting fluid is divided into two streams when through the throttle valve, the former flow through the chip removal channel to the cutting edge, pushing chips into the crumbs port. the latter through the negative pressure channel, formed annular jet, generate a negative pressure suction effect, thereby increasing the pressure difference before and after the pipe interior, namely to enhance the chip removal's power by the negative pressure.(B Wang, 2011) Practice has proved that it played a role in the chip removal process.

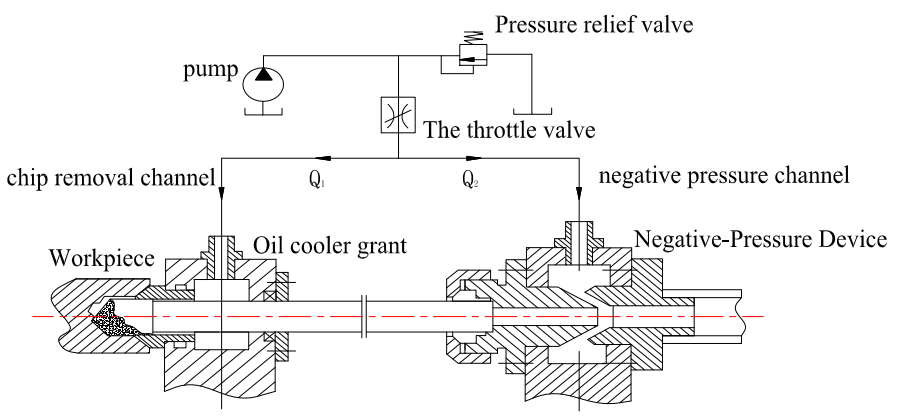

Fig.1 Schematic of Negative pressure chip removal

\section{INFLUENCING FACTORS OF THE} NEGATIVE PRESSURE CHIPS REMOVAL

As shown in Fig.2, the negative pressure pumping chips confluence model, the cross section1-1 is chip removal channel, the diameter is $D_{1}$, flow rate is $Q_{1}$,flow velocity is $v_{1}$, the cross section2-2 is negative pressure channel', the clearance of the jet is $\delta$, flow rate is $Q_{2}$,flow velocity is $v_{2}$. The cross section0-0 is overall channel , the diameter is $D_{0}$, flow rate is $Q_{0}$, flow velocity is $v_{0}$.

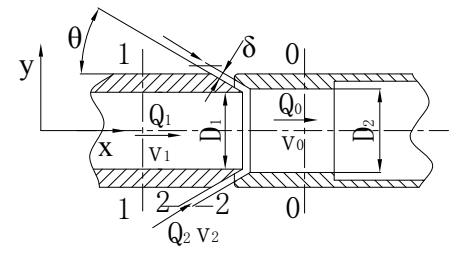

Fig.2 The negative pressure pumping chips confluence model 
As shown in Fig.2, for tributaries and the total flow, by the conservation of mass:

$Q_{0}=Q_{1}+Q_{2}$

The Bernoulli equation of overcurrent through cross section 1-1 and 0-0, and between 2-2 and 0-0:

$\left\{\begin{array}{l}z_{1}+\frac{p_{1}}{\rho g}+\frac{\alpha_{1} v_{1}^{2}}{2 g}=z_{0}+\frac{p_{0}}{\rho g}+\frac{\alpha_{0} v_{0}^{2}}{2 g} \\ z_{2}+\frac{p_{2}}{\rho g}+\frac{\alpha_{2} v_{2}^{2}}{2 g}=z_{0}+\frac{p_{0}}{\rho g}+\frac{\alpha_{0} v_{0}^{2}}{2 g}\end{array}\right.$

The two equations of formula (2) are multiplied by $Q_{1}$ and $Q_{2}$ then adding the two equations. Then use the formula'(1):

$Q_{0}\left(z_{0}+\frac{p_{0}}{\rho g}+\frac{\alpha_{0} v_{0}^{2}}{2 g}\right)=Q_{1}\left(z_{1}+\frac{p_{1}}{\rho g}+\frac{\alpha_{1} v_{1}^{2}}{2 g}\right)+Q_{2}\left(z_{2}+\frac{p_{2}}{\rho g}+\frac{\alpha_{2} v_{2}^{2}}{2 g}\right)$

Equation (3) is the confluence the Bernoulli equation (WL Li, 2009).

And

$Q=v \cdot A$

That $Q_{0}=v_{0} \cdot A_{0}, Q_{1}=v_{1} \cdot A_{1}, Q_{2}=v_{2} \cdot A_{2}$

Wherein, $v_{0}, v_{1}, v_{2}$ for the average speed of the flow cross section; $A_{0}, A_{1}, A_{2}$ as cross-sectional area of each channel.

Therefore, the all parameters of negative pressure chips removal must satisfy the relation (3) and (4).

By conservation of momentum:

$\rho Q_{2} v_{2} \cos \theta+\rho Q_{1} v_{1}=\rho Q_{0} v_{0}$

Assume $Q_{1} / Q_{2}=\beta$

Then

$\Delta v=v_{0}-v_{1}=\frac{v_{2} \cdot \cos \theta-v_{1}}{\beta+1}$

Because

$v_{1}=\frac{Q_{1}}{A_{1}}=\frac{\beta Q_{2}}{\pi\left(D_{1} / 2\right)^{2}}=\frac{4 \beta Q_{2}}{\pi D_{1}^{2}}$

$v_{2}=\frac{Q_{2}}{A_{2}}=\frac{Q_{2}}{\pi D_{1} \delta}$

$\Delta p_{n}=\Delta p^{\prime}-\Delta p=\frac{\rho}{2}\left(\Delta v^{2}+2 v_{1} \cdot \Delta v\right)$

$\Delta p_{n}$ is the negative pressure value. Then

$\Delta p_{n}=\frac{\rho Q_{2}^{2}}{2(\beta+1)^{2}}\left(\frac{\cos \theta}{\pi D_{1} \delta}-\frac{4 \beta}{\pi D_{1}^{2}}\right)^{2}+\frac{4 \rho \beta Q_{2}^{2}}{\beta+1}\left(\frac{D_{1} \cos \theta}{\delta}-4 \beta\right)$

In the formula, $\theta$ is the spray angle of the jet nozzle.

\section{SIMULATION ANALYSIS OF NEGATIVE- PRESSURE CHIP REMOVING DEVICE}

\subsection{Model building}

Assumed $\quad D_{1}=17 \mathrm{~mm}, \quad \delta=0.45 \mathrm{~mm}, \quad \theta=30^{\circ}$, $D_{2}=D_{1}+2 \delta \cos \theta$, three-dimensional model of negative pressure Chip Removing device as shown in Figure 3.

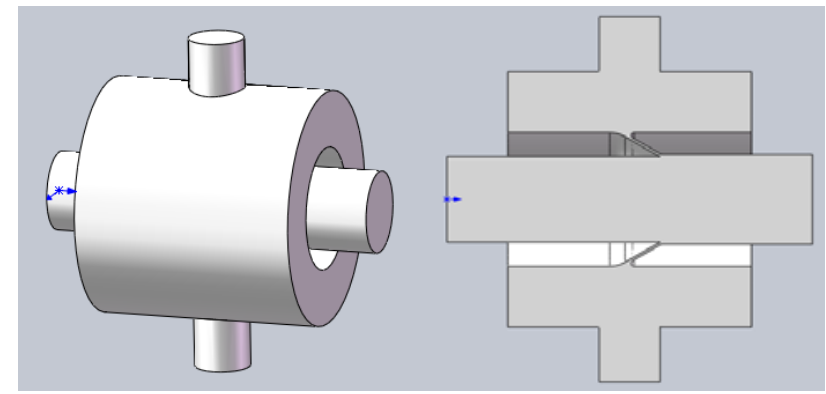

Fig.3 The three-dimensional model of flow field in the negative pressure chips removal

Two-dimensional model and A partially enlarged view shown in Figure 4.

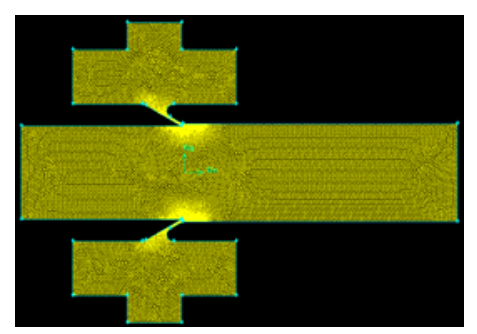

(a)Two-dimensional model

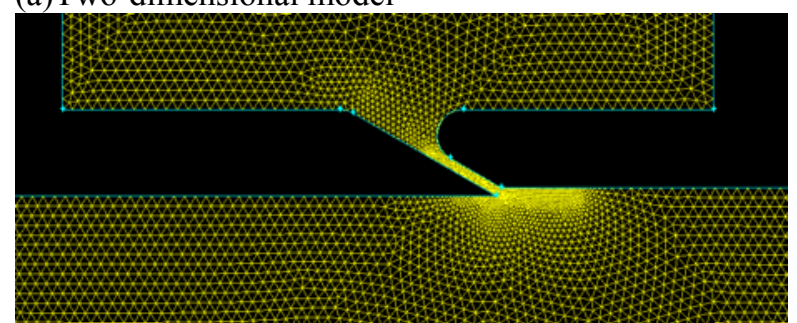

(b) Partially enlarged view

Fig.4 The two-dimensional model

\subsection{Solver settings}

Established the fluid material to cure the cutting fluid (Sulfur-Liquid), its density is $2000 \mathrm{~kg} / \mathrm{m}^{3}$, viscosity is $1.72 \times 10^{-5} \mathrm{~kg} / \mathrm{m} \cdot \mathrm{s}$; set the boundary conditions, make flow rate in chip removal channel as $92 \mathrm{~L} / \mathrm{min}$, the flow rate in the negative pressure channel is $46 \mathrm{~L} / \mathrm{min}$, that is: the velocity of chip removal channel inlet is $6.76 \mathrm{~m} / \mathrm{s}$, the jet port speed is $31.9 \mathrm{~m} / \mathrm{s}$, Convergence criteria for selecting the differential equation of the continuity equation to calculate the difference between the two sides is less than 0.0001 prevail.

\subsection{Simulation results}

By simulation calculation, the overall pressure nephogram and the negative pressure zone radial section full pressure scatterplot are shown in Fig. 5 (a), (b), (c). 


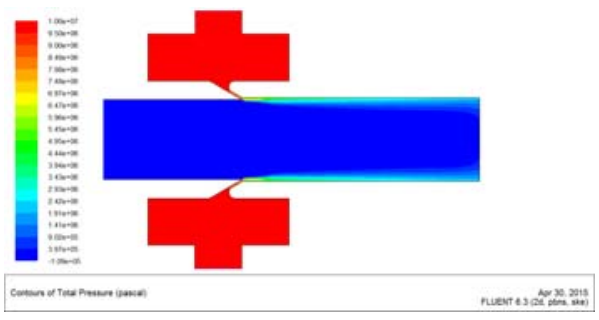

(a) Contours of total pressure

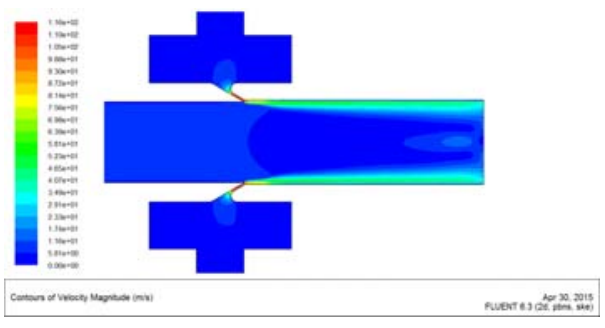

(b) Contours of velocity magnitude

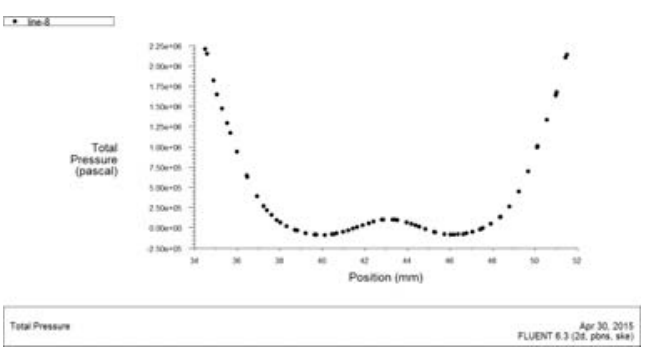

(c) Total Pressure scatterplot in the negative pressure zone radial section

Fig.5 Simulation results

\section{SIMULATION ANALYSIS WITHOUT NEGATIVE-PRESSURE CHIP REMOVING DEVICE}

Assume the diameter of the chip removal channel $D_{1}=17 \mathrm{~mm}$. Established the fluid material to cure the cutting fluid (Sulfur-Liquid), its density is $2000 \mathrm{~kg} / \mathrm{m}^{3}$, viscosity is $1.72 \times 10^{-5} \mathrm{~kg} / \mathrm{m} \cdot \mathrm{s}$; set the boundary conditions, make flow rate in chip removal channel as $92 \mathrm{~L} / \mathrm{min}$, convergence criteria for selecting the differential equation of the continuity equation to calculate the difference between the two sides is less than 0.0001 prevail.

By simulation calculation, the overall pressure nephogram, the speed nephogram are shown in Fig.6 (a), (b).

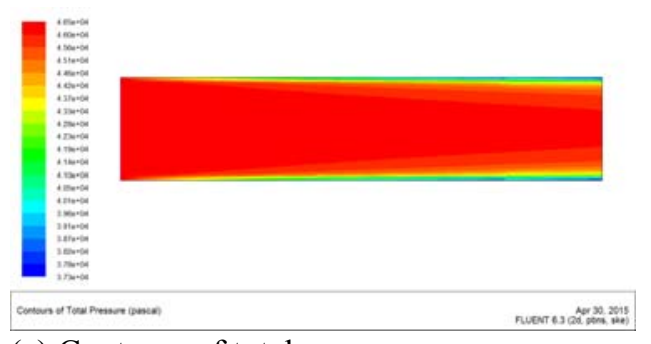

(a) Contours of total pressure

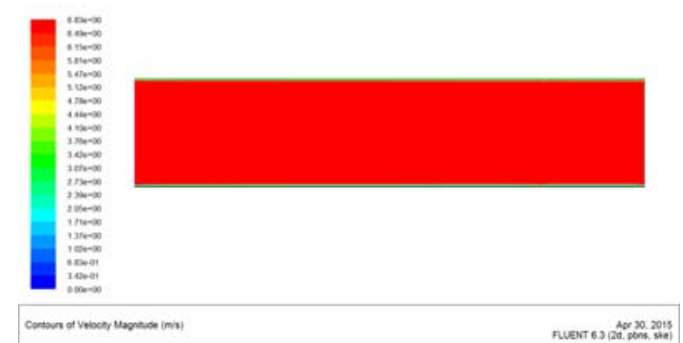

(b) Contours of velocity magnitude

Fig.6 Simulation results

\section{RESULT ANALYSIS}

Comparative Fig.6 and 7, it can be seen, without negative-pressure chip removing device, there is only a thrust on the chip from the entrance of the channel, and the pressure is $4.6 \times 10^{4} \mathrm{~Pa}$. The negativepressure chip removing device can produce a negative pressure, when The flow rate in the negative pressure channel is $46 \mathrm{~L} / \mathrm{min}$, It produces a negative pressure is $1.5 \times 10^{5} \mathrm{~Pa}$, suction force directly on the chip, plus thrust from the entrance of the channel, it enables the chip to accelerate discharged with cutting fluid, more conducive to chip removing smoothly.

\section{ACKNOWLEDGEMENTS}

This work was financially supported by the National Natural Science Foundation of China (51175482)and International S\&T Cooperation Program of China (2013DFA70770).

\section{REFERENCES}

B Wang. Simulation of negative chip DF deep hole drilling systems and experimental study [D]. China: Xi'an University of Technology, 2011.

J Wang. Modern deep processing technology [M].China: Harbin Institute of Technology Press, 2005: 1-433.

Jih-Hua Chin, Jang-Shyong Wu. Mathematical models and experiments for chip signals of single-edge deep hole drilling [J]. International Journal of Machine Tools and Manufacture, 1993, 33(3): 507-519.

NJ Zhou. Engineering Fluid Mechanics foundation [M]. China: Machinery Industry Press, 2012: 1-260.

SF Shi. The study of Near Dry Deep hole processing system architecture based on FLUENT Software [D]. China: North University, 2012.

WL Li.Modeling and Simulation of deep hole processing chip mechanism DF systems [D]. China: North University, 2009.

SQ Wang, Zhu L, ZF Liu, H Peng.Deep hole processing tech nology [M].China: Northwestern University Press, 2003: 1239.

YD Zhang,L Chen,WH Li.The influencing factors of the effect of negative pressure pumping crumbs in deep hole processing[J]. 2005 Chinese Mechanical Engineering Society Proceedings. 2005. 